\title{
Lipopolysaccharide induces intestinal glucocorticoid synthesis in a TNF $\alpha$-dependent manner
}

\author{
Mario Noti, $*$ Nadia Corazza, $*$ Gérald Tuffin, ${ }^{\dagger}$ Kristina Schoonjans, $^{\ddagger}$ \\ and Thomas Brunner*, \\ *Division of Experimental Pathology, Institute of Pathology, University of Bern, Bern, Switzerland; \\ 'University of Applied Sciences Northwestern Switzerland, School of Life Sciences, Muttenz, \\ Switzerland; and 'École Polytechnique Fédérale de Lausanne, Laboratory of Integrative Systems \\ and Physiology, Lausanne, Switzerland
}

\begin{abstract}
Stringent control of immune responses in the intestinal mucosa is critical for the maintenance of immune homeostasis and prevention of tissue damage, such as observed during inflammatory bowel disease. Intestinal epithelial cells, primarily thought to form a simple physical barrier, critically regulate intestinal immune cell functions by producing immunoregulatory glucocorticoids on T-cell activation. In this study we investigated whether stimulation of cells of the innate immune system results in the induction of intestinal glucocorticoids synthesis and what role TNF- $\alpha$ plays in this process. Stimulation of the innate immune system with lipopolysaccharide (LPS) led to an upregulation of colonic steroidogenic enzymes and the induction of intestinal glucocorticoid synthesis. The observed induction was dependent on macrophage effector functions, as depletion of macrophages using clodronate-containing liposomes, but not absence of $T$ and $B$ cells, inhibited intestinal glucocorticoid synthesis. LPS-induced glucocorticoid synthesis was critically dependent on TNF- $\alpha$ as it was significantly decreased in TNF- $\alpha$-deficient animals. Both TNF receptor-1 and -2 were found to be equally involved in LPS- and T-cellinduced intestinal GC synthesis. These results describe a novel and critical role of TNF- $\alpha$ in immune cellinduced intestinal glucocorticoid synthesis.-Noti, M., Corazza, N., Tuffin, G., Schoonjans, K., Brunner, T. Lipopolysaccharide induces intestinal glucocorticoid synthesis in a TNF $\alpha$-dependent manner.
\end{abstract}

Key Words: inflammatory bowel disease $\cdot$ tumor necrosis factor $\alpha \cdot$ intestinal epithelial cells $\cdot$ macrophages $\cdot$ TNF receptors

The MUCOSAL SURFAcE of THE gastrointestinal tract is in constant contact with luminal factors and is challenged by potentially invasive and pathogenic microorganisms. Inappropriate activation of immune cells from either the innate or the adaptive immune system may result in chronic inflammatory reactions and associated tissue destruction, e.g., as observed in inflammatory bowel disease (IBD) (1-3). Therefore, local immune responses have to be tightly regulated (4). Various regulatory mechanisms have been described previously contribut- ing to the maintenance of immune homeostasis in the intestinal mucosa. These include, among others, regulatory $T$ cells and the release of immunosuppressive cytokines (5-7)

Glucocorticoids (GCs) are steroid hormones with important immunoregulatory activities (8). GCs regulate immune cells by either suppressing or stimulating various cellular processes $(9,10)$. Best known are their NFкB-inhibiting properties and subsequent effects on the production of proinflammatory cytokines $(11,12)$. The adrenal glands are a well-characterized source of GCs and produce these hormones in large quantities in response to emotional, physical, and immunological stress (13). Yet there is increasing evidence for extraadrenal GC synthesis in other organs and tissues (14). We recently described that epithelial cells of the intestinal crypts are an important source of extra-adrenal GC synthesis and produce these hormones locally on T-cell stimulation. Critically, we showed that absence of intestinal GC synthesis leads to increased activation of intestinal antigen-specific $\mathrm{T}$ cells during viral infections, confirming the immunosuppressive role of locally produced intestinal GCs (15)

The factors that regulate intestinal GC synthesis are still poorly explored. Although we have identified the nuclear receptor and transcription factor liver receptor homologue-1 (LRH-1, NR5a2) as a critical regulator of steroidogenic enzyme expression in intestinal epithelial cells, little is known about the factors that are released from immune cells triggering intestinal GC synthesis (16). Because stimulation of cells of the adaptive immune system induces intestinal GC synthesis, we aimed at investigating whether activation of cells of the innate immune system similarly promotes intestinal steroidogenesis. We therefore injected mice i.p. with lipopolysaccharide (LPS) to stimulate various myeloid effector cells and analyzed its consequences on intestinal GC synthesis. LPS was found to be a most potent inducer of intestinal GC synthesis. LPS-induced intestinal steroido-

\footnotetext{
${ }^{1}$ Correspondence: Division of Experimental Pathology, Institute of Pathology, University of Bern, Murtenstrasse 31, 3010 Bern, Switzerland. E-mail: tbrunner@pathology.unibe.ch doi: $10.1096 /$ fj. $09-140913$
} 
genesis appeared to be at least partially dependent on the activation of macrophages, as depletion of macrophages by clodronate-encapsulated liposomes substantially reduced GC synthesis. In marked contrast, absence of $\mathrm{T}$ cells did not affect LPS-induced intestinal GC synthesis. TNF- $\alpha$ appeared to be a critical mediator in LPS- and T-cell-induced intestinal steroidogenesis, as local GC synthesis was abrogated in TNF- $\alpha$ - or TNF receptor (TNFR)-deficient mice. Interestingly, TNFR1 and 2 equally contributed to $\mathrm{TNF} \sim \alpha$-mediated intestinal GC synthesis. This study demonstrates for the first time that LPS induces intestinal GC synthesis in a TNF- $\alpha$ dependent manner.

\section{MATERIALS AND METHODS}

\section{Animals}

All animal experiments were performed in compliance with the laws and guidelines of the State of Bern. Animals were breed and kept in the Central Animal Facility of the Department of Medicine, University of Bern, and group-housed with free access to food and water. TNF- $\alpha^{-1-}$, TNFR $1^{-1-}$, TNFR $2^{-1-}, \mathrm{IL} 6^{-1-}$, Rag2 ${ }^{-1-}$, and LRH- $1^{+/-}$mice under the $\mathrm{C} 57 \mathrm{Bl} / 6$ background have been described previously (17-19). Mice were used for experiments between 7 and $10 \mathrm{wk}$ of age.

\section{In vivo administration of LPS and anti-CD3e}

Animals were treated with LPS (from Escherichia coli 055:B5, $100 \mu \mathrm{g} /$ animal; Sigma-Aldrich, St Louis, MO, USA) or antiCD3e (20 $\mu \mathrm{g} /$ animal) (15) by i.p. injection. After $3 \mathrm{~h}$ animals were sacrificed, and colonic tissue was isolated and either snap-frozen for further RNA isolation or cultured ex vivo for analysis of GC synthesis (15).

\section{Detection of corticosterone synthesis in intestinal organ cultures}

The entire large intestine was opened longitudinally, washed with $\mathrm{PBS} / 2 \%$ charcoal-stripped horse serum, and cut into 5-mm-long pieces. One-third of the entire colon was sampled and snap-frozen for further RNA isolation. Remaining colonic tissue was randomly distributed to 2 wells of a 24-well plate and cultured for $6 \mathrm{~h}$ at $37^{\circ} \mathrm{C}$ in Iscove's modified Dulbecco's medium (IMDM) and in the presence of the specific GC inhibitor metyrapone $(200 \mu \mathrm{g} / \mathrm{ml}$; Sigma-Aldrich). Cell-free supernatant was harvested, and corticosterone concentrations were measured using a radioimmunoassay. Results are expressed as ng corticosterone per gram of tissue and shown as the difference of samples cultured without and with metyrapone (metyrapone-blockable corticosterone synthesis) to correct for variable contamination with serum glucocorticoids (15). The efficacy of inhibition of corticosterone synthesis in colonic organ cultures by metyrapone is illustrated in Supplemental Fig. 1.

\section{Macrophage depletion by clodronate-encapsulated liposomes}

Macrophages were depleted in vivo as described previously (20). Briefly, clodronate (kindly provided by Roche Pharmaceuticals, Mannheim, Germany) was encapsulated in liposomes composed of $50 \mu \mathrm{M}$ distearoyl-phosphatidylglycerol
(DSPG; Avanti, Birmingham, AL, USA), $100 \mu \mathrm{M}$ cholesterol (Sigma-Aldrich), and $150 \mu \mathrm{M}$ of 1,2-distearoyl-sn-glycero-3phosphocholine (DSPC; Avanti) by reverse-phase evaporation technique. As negative control, white liposomes (WLs) were generated in parallel. Mice were injected i.v. with $100 \mu \mathrm{M}$ of lipid/kg clodronate-liposomes, WLs, or PBS. After $48 \mathrm{~h}$, depletion of macrophages in spleen, liver, and in testine was confirmed by immunohistochemistry using an F4/80 macrophage marker antibody.

\section{Real-time PCR}

Colonic tissue was homogenized and lysed in TRI-reagent (Sigma-Aldrich) for further RNA isolation. RNA was DNase treated and reverse transcribed using a high-capacity RT kit from Applied Biosystems (Foster City, CA, USA). RT-PCR was performed using Quantitect primer assays (Qiagen; Valencia, CA, USA) for the steroidogenic enzymes CYP11B1 and CYP11A1, as described previously (16). GAPDH or Villin was used to normalize expression levels.

\section{Cytokine ELISA for TNF- $\alpha$ and IL-6}

Sera of animals treated with white liposomes as control or animals treated with clodronate-encapsulated liposomes were sampled, and TNF- $\alpha$ and IL-6 concentrations were measured using matched antibody pair ELISA (BioLegend, San Diego, CA, USA).

\section{Statistics}

Results are expressed as means \pm sD. Differences between groups were analyzed by unpaired Student's $t$ test. Values of $P<0.05$ were considered statistically significant.

\section{RESULTS}

\section{LPS induces intestinal GC synthesis in a T-cell-independent manner}

We have previously shown that activation of $\mathrm{T}$ cells by injection of an agonistic anti-CD3 $\varepsilon$ antibody results in rapid induction of steroidogenic enzymes and associated GC synthesis in the intestinal epithelium $(15,16)$. To investigate whether intestinal GC synthesis could be triggered not only by cells of the adaptive immune system, e.g., T cells, but also by activation of the innate immune system, we injected $\mathrm{C} 57 \mathrm{Bl} / 6$ mice with a single dose of LPS, or anti-CD $3 \varepsilon$ antibody as positive control, for $3 \mathrm{~h}$ and analyzed the induction of local steroidogenesis in colonic tissue. Injection of anti-CD $3 \varepsilon$ led to a significant increase in the expression of the steroidogenic enzymes CYP11B1 (11Bhydroxylase) (Fig. 1E) and CYP11A1 (P450scc) (Fig. $1 D$; data added in proof), and associated intestinal GC synthesis (Fig. 1F). Critically, GC synthesis in ex vivo cultured colonic tissue was blockable by the GC synthesis inhibitor metyrapone, confirming that GCs measured were bona fide in situ produced (15). Interestingly, LPS strongly induced CYP11B1 (Fig. $1 B$ ) and CYP11A1 (Fig. 1A; data added in proof) mRNA expression levels and colonic GC synthesis (Fig. 1G). Anti-CD3 

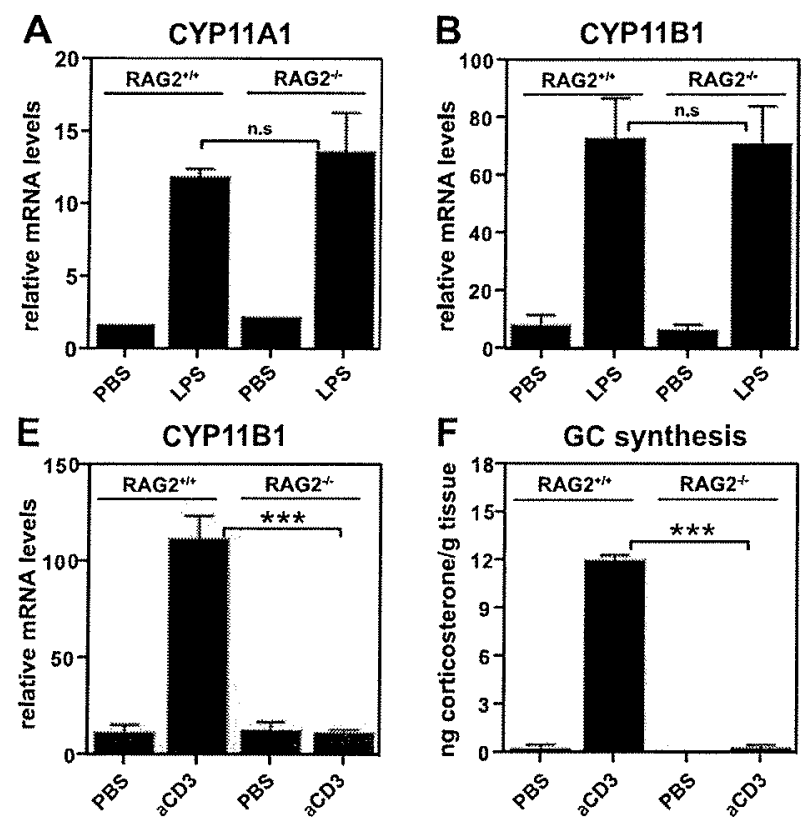

injection seemed to be a more potent stimulus for the induction of intestinal GC synthesis than LPS (Fig. 1C, $F$ ), although both triggers were equally effective in promoting the release of GCs from the adrenal glands into circulation (Supplemental Fig. 2).

As we previously characterized T-cell-dependent induction of intestinal steroidogenesis, we further assessed whether LPS-induced GC synthesis was also dependent on the presence of $\mathrm{T}$ cells. T-cell (and B-cell)-deficient RAG2 $2^{-1-}$ mice were thus injected with anti-CD3 $\varepsilon$ antibody or LPS, and colonic GC synthesis was analyzed. As expected, anti-CD3e antibody-induced GC synthesis as well as induction of CYP11B1 expression was completely blocked in the lymphopenic $\mathrm{RAG2}^{-/-}$mice (Fig. $1 E, F$ ). In marked contrast, no inhibition was observed in intestinal steroidogenesis when $\mathrm{RAG}^{-1-}$ mice were treated with LPS (Fig. $1 B, C$ ). These findings indicate that LPS promotes colonic GC synthesis in a T-cell-independent manner.
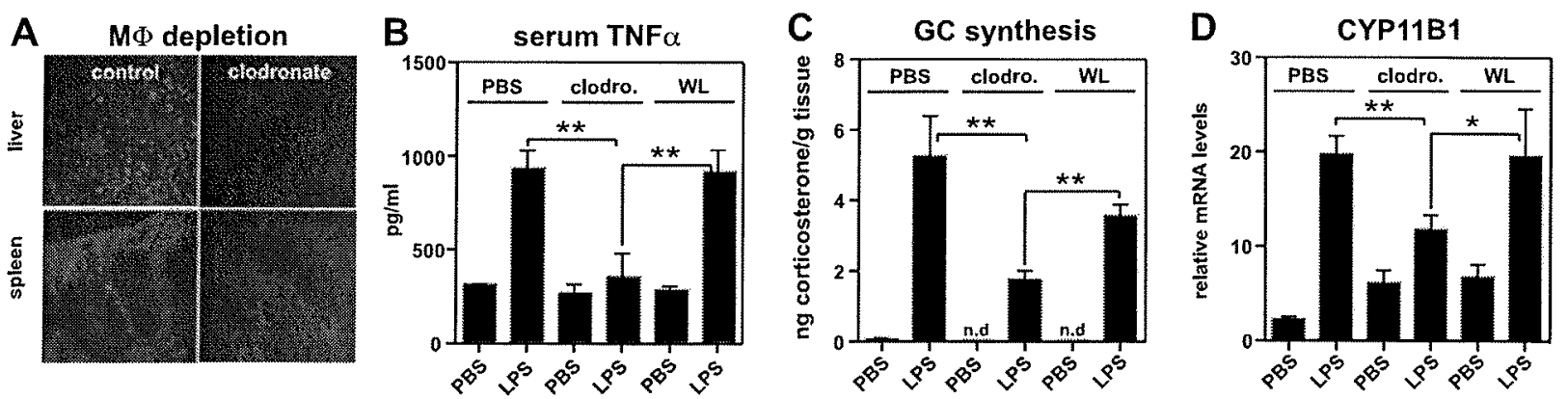

Figure 2. Role of macrophages in LPS-induced intestinal GC synthesis. Animals were injected i.v. with clodronate-encapsulated liposomes or control liposomes (WL) 48 and $24 \mathrm{~h}$ prior to LPS treatment. A) Depletion of macrophages in liver and spleen, confirmed by macrophage-specific F4/80 immuno-fluorescence. B) Serum protein levels of TNF- $\alpha$ in macrophage-depleted animals (clodro.) and controls (PBS, WL) after LPS treatment. $C, D$ ) Colonic CYP11B1 mRNA expression $(C)$ and associated intestinal GC synthesis $(D)$ in macrophage-depleted and control-treated animals after LPS administration. Data are means \pm SD; $n=5$ /group. $* P<0.05 ; * * P<0.01$. n.d., not detected. 

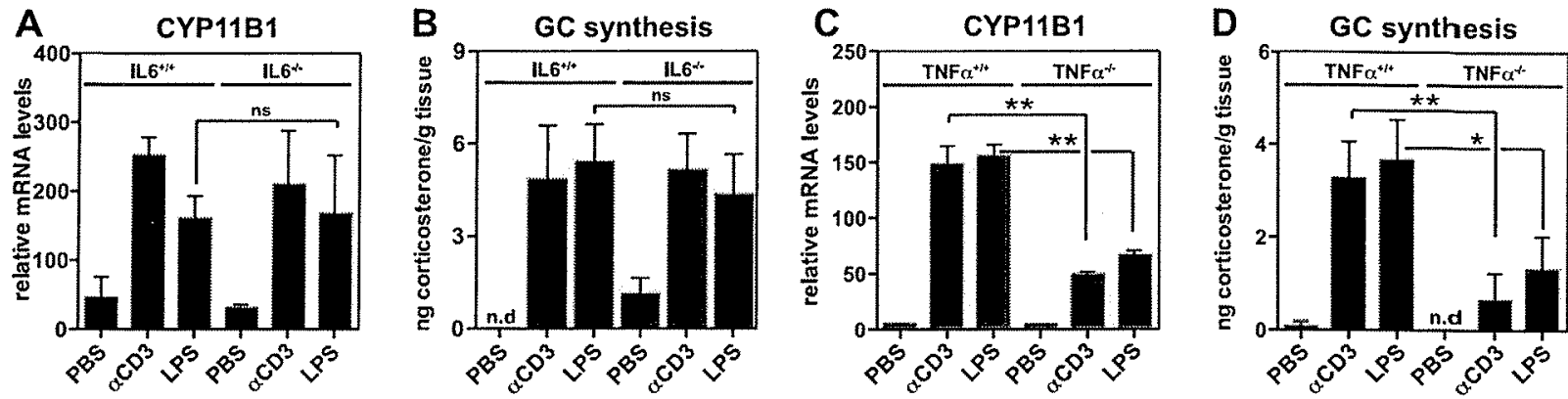

Figure 3. TNF- $\alpha$ but not IL-6 is critical for intestinal GC synthesis. Wild-type, TNF- $\alpha$-, and IL-6-deficient animals were injected i.p. with LPS or anti-CD $3 \varepsilon$ for 3 h. $A, B)$ Colonic CYP11B1 mRNA expression levels $(A)$ and associated GC synthesis $(B)$ in colonic organ cultures of LPS- or anti-CD3E-treated wild-type and IL-6-deficient animals. $C, D$ ) Colonic CYP11B1 mRNA expression levels $(C)$ and associated intestinal GC synthesis $(D)$ in TNF- $\alpha$-deficient animals after LPS or anti-CD3 $\varepsilon$ treatment. Results are means \pm SD; $n=6 .{ }^{*} P<0.05 ; * * P<0.01$. n.d., not detected.

IL-6 levels, in agreement with the notion that macrophages are a major source of these proinflammatory cytokines (Fig. 2B; IL-6 levels not shown).

Although LPS-induced intestinal GC synthesis in mice treated with WLs did not significantly differ from PBStreated control mice, we observed a substantial inhibition of colonic GC production in mice treated with clodronate-encapsulated liposomes (Fig. 2C). Depletion of macrophages resulted in reduced LPS-induced CYP11B1 expression levels compared to control treated mice (Fig. $2 D)$. These findings indicate that macrophages substantially contribute to LPS-induced intestinal GC synthesis.

TNF- $\alpha$ but not IL 6 is critical for intestinal GC synthesis

Systemic administration of LPS results in massive production and secretion of the proinflammatory cytokines TNF- $\alpha$ and IL 6 by activated macrophages, as described above (22). As macrophage depletion resulted in clearly reduced serum levels of TNF- $\alpha$ and IL-6 on LPS stimulation (Fig. 2B), we investigated the role of TNF- $\alpha$ and IL-6 on the induction of intestinal steroidogenesis. IL-6- and TNF- $\alpha$-deficient mice were injected with PBS control, anti-CD3E, or LPS, and intestinal steroidogenesis was assessed. Absence of IL-6 did not alter the induction of intestinal GC synthesis in response to both anti-CD3 $\varepsilon$ and LPS treatment (Fig. 3A, $B)$. In marked contrast, absence of TNF- $\alpha$ resulted in significantly reduced anti-CD3E- and LPS-induced colonic CXP11B1 mRNA expression levels and associated corticosterone synthesis (Fig. $3 C, D$ ). These findings support the notion that both T-cell- and macrophageinduced intestinal GC synthesis proceeds via TNF- $\alpha$.

\section{Role of TNFR1 and TNFR2 in intestinal GC synthesis}

TNF- $\alpha$ activates cells via 2 different receptors, TNFR1 and TNFR2 (23, 24). As these 2 receptors engage similar but also different signaling pathways, we aimed at investigating the respective roles of TNFRI and TNFR2 in LPS-induced and TNF- $\alpha$-mediated intestinal GC synthesis. Wild-type, TNFR1 ${ }^{-1-}$, and TNFR2 ${ }^{-1-}$ mice were injected with LPS, or anti-CD3E as control, and colonic GC synthesis and CYP11B1 expression were analyzed. Although in wild-type animals LPS and antiCD3 $\varepsilon$ strongly induced intestinal corticosterone production and expression of CYP11B1, both GC synthesis and associated steroidogenic enzyme expression were significantly reduced in $\mathrm{TNFR}^{-1-}$ and $\mathrm{TNFR}^{-1-}$ mice (Fig. 4). Thus, both TNFR1 and TNFR2 critically contribute to TNF- $\alpha$-mediated colonic steroidogenesis.
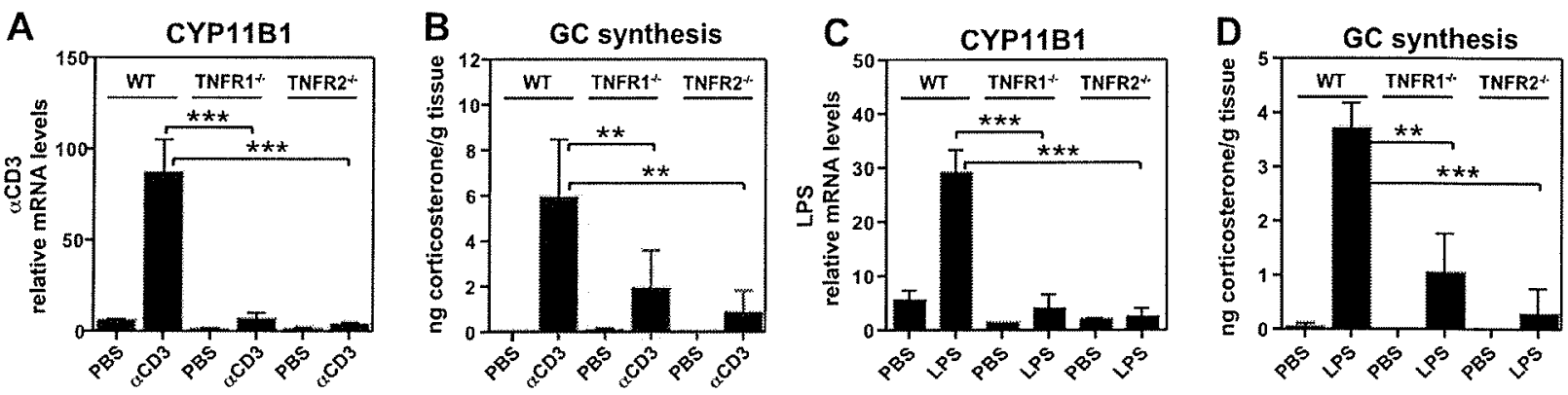

Figure 4. Role of TNFR1 and TNFR2 in intestinal GC synthesis. Wild-type, TNFR1-, and TNFR2-deficient animals were injected i.p. with LPS or anti-CD $3 \varepsilon$ for $3 \mathrm{~h}$. $A, B$ ) Colonic CYP11Bl mRNA expression levels $(A)$ and associated intestinal corticosterone synthesis $(B)$ after anti-CD3e treatment. $C, D$ ) Colonic CYPl1Bl mRNA expression levels $(C)$ and GC synthesis $(D)$ after treatment with LPS. Results are means $\pm \mathrm{sD} ; n=5$ /group. $* * P<0.01 ; * * *<0.005$. 
Different regulation of $\mathrm{T}$-cell- and macrophage-induced intestinal steroidogenesis

Steroidogenesis critically depends on transcription factors of the nuclear receptor family by regulating the expression of steroidogenic enzymes (25). While in the adrenal glands steroidogenic factor-1 (SF-1, NR5a1) represents the key regulator of glucocorticoid synthesis, we recently described that in the intestinal mucosa its function is replaced by the close homologue LRH-1 (NR5a2). Consequently, anti-CD3e-induced colonic GC synthesis was impaired in LRH-1 haplodeficient mice (18; Fig. 5). We thus aimed at investigating the respective role of LRH-1 in LPS-induced intestinal GC synthesis. Surprisingly, we observed that LRH-1 haplodeficient mice expressed even higher levels of CYP11B1 mRNA and showed a tendency toward higher corticosterone levels in response to LPS than wild-type mice (Fig. 5). This finding indicates a differential requirement for LRH-1 in the regulation of colonic GC synthesis.

\section{DISCUSSION}

In vivo stimulation of macrophages with LPS or T cells with anti-CD3 antibody, respectively, rapidly induces in testinal epithelial cell damage characterized by loss of villi and enterocyte apoptosis $(26,27)$. T-cell and macrophage activation also results in massive release of the proinflammatory cytokine TNF- $\alpha$, which contributes to the damage of the epithelial layer. The detrimental effects of TNF- $\alpha$ are reflected in the breakdown of the integrity of the intestinal epithelial layer, characterized by intestinal epithelial cell apoptosis $(27,28)$. The proinflammatory and pathogenic role of TNF- $\alpha$ is particularly highlighted by the effectiveness of humanized TNF- $\alpha$ neutralizing antibodies, such as Infliximab, in the treatment of IBD, leading to a profound clinical improvement of the disease pathology $(29,30)$.
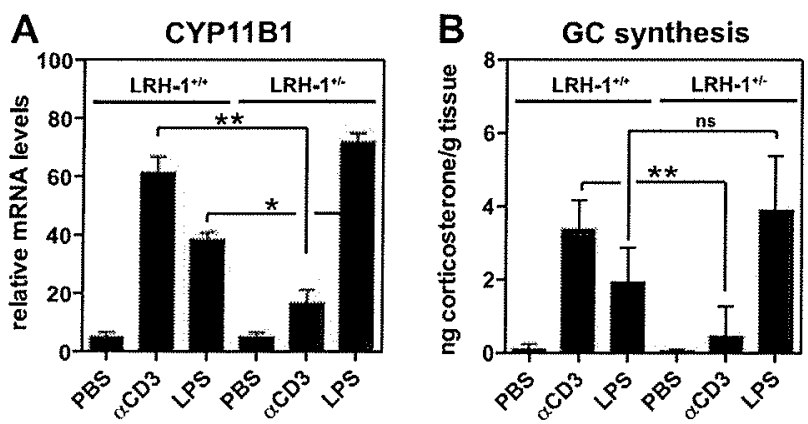

Figure 5. Differential role of LRH-1 in T-cell- and macrophageinduced intestinal steroidogenesis. Wild-type $\left(\mathrm{LRH}-1^{+/+}\right)$and LRH1 haplodeficient (LRH-1 ${ }^{+/-}$) animals were injected i.p. with LPS or anti-CD3 $\varepsilon$ for $3 \mathrm{~h}$. Colonic CYP11B $1 \mathrm{mRNA}$ expression levels $(A)$ and associated intestinal corticosterone synthesis (B) after LPS or anti-CD $3 \varepsilon$ treatment. Results are means $\pm \mathrm{SD}$; $n=5$ /group. $* P<0.05 ; * * P<0.01$. ns, not significant.
Apart from the well-known proinflammatory properties of TNF- $\alpha$ there is now emerging evidence for an antiinflammatory role of TNF- $\alpha$ (31-33). In part, antiinflammatory properties of TNF- $\alpha$ might be attributed to its apoptosis-inducing and/or apoptosis-modulating activities in immune cells. Our own results suggest, however, that induction of local steroidogenesis may count as a novel TNF- $\alpha$-induced antiinflammatory mechanism. We herein show that intestinal GC synthesis is markedly increased on activation of the innate and adaptive immune system. Furthermore, we identified TNF- $\alpha$ as a critical regulator of intestinal GC synthesis because in the absence of TNF- $\alpha$ intestinal GC synthesis was drastically impaired on stimulation with LPS or anti-CD3e. Thus, TNF- $\alpha$ appears to have a novel, rather paradoxical, antiinflammatory activity in the intestinal mucosa via the induction of GC synthesis in intestinal epithelial cells.

Notably, so far all previously described antiinflammatory properties of TNF- $\alpha$ have been attributed to their activity on $T$ cells (31-33). Our findings now provide evidence for an alternative pathway via the induction of intestinal steroidogenesis in nonhematopoietic cells. Although in vitro experiments with intestinal epithelial cell lines prove that TNF- $\alpha$ can directly induce the expression of steroidogenic enzymes (unpublished results), the exact mode of action and associated mechanisms of TNF- $\alpha$-induced or TNF- $\alpha$-mediated intestinal GC synthesis remain to be elucidated. Our previous data have clearly shown that intestinal steroidogenesis critically contributes to the maintenance of intestinal immune homeostasis $(15,18)$. Although a direct inhibition of immune cells is very likely, indirect effects should also be considered. Thus, local intestinal GC synthesis may counterbalance the deleterious effects of TNF- $\alpha$ in two ways: 1) an increase in barrier resistance by promoting the expression of tight junction proteins (34) and 2) by dampening overwhelming immune responses and activation of associated immune cells, which might be initiated by unrestrained access to luminal factors after epithelial barrier disruption (15, 18). Thus, although TNF- $\alpha$ is involved in the disruption of the epithelial barrier integrity, it is also a prime sensor cytokine involved in monitoring and restoring intestinal epithelial barrier function, e.g., via the induction of GC synthesis. As TNF- $\alpha$ production is not restricted to macrophages and $\mathrm{T}$ cells, but may also be induced in intestinal epithelial cells (35), it is feasible to believe that this regulatory system may even work in an epithelial layer autonomous manner.

Intraperitoneal application of LPS immediately activates peripheral macrophages via Toll-like receptor 4 (TLR4) and associated coreceptor subunits leading to induced expression of proinflammatory cytokines, such as TNF- $\alpha$, IL-1 $\beta$, and IL-6 (36). In marked contrast, resident intestinal macrophages usually lack expression of TLR4, CD 14, and other activating receptors, such as TREM-1, under healthy conditions. The lack of triggering receptors seen in intestinal macrophages leads to a relative hyporesponsiveness toward LPS and therefore inhibits a downstream immune response by inappropri- 
ate activation of NFKB (37-39). This notion would suggest that peripheral rather than local macrophages are the relevant targets of systemic LPS treatment and source of TNF- $\alpha$. Indeed, clodronate liposome treatment resulted in both a depletion of macrophages in spleen and liver as well as reduced TNF- $\alpha$-mediated intestinal GC synthesis (Fig. 2A, $C$ ). In contrast, depletion of intestinal macrophages after clodronate liposome treatment was less obvious (data not shown). However, intestinal resident macrophages up-regulate coreceptors and TLRs under inflammatory conditions, e.g., in IBD $(40,41)$. Thus, LPS may first trigger systemic macrophages and later also sensitized resident intestinal macrophages. In line with this is our observation that LPS injection leads to TNF- $\alpha$ and IL -6 mRNA expression in colonic tissue (Fig. $2 B$, and data not shown), indicating that local macrophages also become activated by LPS and participate in the induction of local GC synthesis. Therefore, LPS-induced and TNF- $\alpha$-mediated local GC synthesis may contribute to local immune homeostasis as a negative feedback mechanism via the inhibition of NFKB signaling pathways. Because increased inflammation will also result in increased exposure to LPS and TNF- $\alpha$, e.g., after disruption of the epithelial barrier, the local induction of antiinflammatory GCs by these inflammatory signals may comprise a critical adaptation to limit overwhelming and destructive immune responses in the gastrointestinal tract.

We have previously shown the importance of the orphan nuclear receptor LRH-1 in the regulation of intestinal steroidogenesis (16). Interestingly, although T-cell-mediated intestinal GC synthesis is clearly reduced in LRH-1 haplodeficient animals (18) (Fig. $5 B$ ), LPS-induced intestinal steroidogenesis seems not to be dependent on the transcription factor LRH-1 (Fig. $5 B$ ). This is a rather surprising finding because we demonstrated that TNF- $\alpha$ is involved in both LPS- and antiCD3-induced intestinal GC synthesis. Furthermore, TNF- $\alpha$ signaling has been shown to induce the expression of LRH-1 in the liver leading to recovery from obstructive cholestasis (42). Given the differential role of LRH-1 in T-cell- $v s$. macrophage-mediated intestinal GC synthesis, it is likely that additional yet to be identified signals and transcription factors may be involved in LPS-induced intestinal GG synthesis. Furthermore, these findings illustrate a differential adaptation of the intestinal epithelium to signals from the adaptive and the innate immune system.

In conclusion, we identified TNF- $\alpha$ as a sensor of intestinal immune responses and a master switch in the regulation of intestinal GC synthesis in response to activation of the innate and adaptive immune system. Furthermore, our data indicate a novel antinflammatory property of $\mathrm{TNF}-\alpha$ by the induction of local intestinal steroidogenesis.

The authors thank the T.B. and K.S. lab for technical help. The authors further thank Christoph Mueller (University of Bern, Bern, Switzerland) for providing $\mathrm{RAG}^{-1-}, \mathrm{TNF}_{-} \alpha^{-/-}$, TNFR1 ${ }^{-1}$, and TNFR2 ${ }^{-1-}$ animals and Manfred Kopf (Eidgenössische Technische Hochschule Zürich, Zurich, Swit- zerland) for the IL-6 $6^{-1-}$ mice. This work was supported by grants from the Crohn's and Colitis Foundation of America, the Swiss National Science Foundation, and Oncosuisse to T.B. The authors declare no conflicts of interest.

\section{REFERENCES}

1. Cario, E. (2008) Innate immune signalling at intestinal mucosal surfaces: a fine line between host protection and destruction. Curr. Opin. Gastroenterol. 24, 725-732

2. Lee, J., Gonzales-Navajas, J. M., and Raz, E. (2008) The "polar izing-tolerizing" mechanism of intestinal epithelium: its relevance to colonic homeostasis. Semin. Immunopathol. 30, 3-9

3. McGuckin, M. A., Eri, R., Simms, L. A., Florin, T. H., and Radford-Smith, G. (2009) Intestinal barrier dysfunction in inflammatory bowel diseases. Inflamm. Bowel Dis. 15, 100-113

4. Boirivant, M., Amendola, A, and Butera, A. (2008) Intestinal microflora and immunoregulation. Mucosal. Immunol. 1 (Suppl. 1), $\$ 47-\$ 49$

5. Salzman, N. H., Underwood, M. A., and Bevins, C. L. (2007) Paneth cells, defensins, and the commensal microbiota: a hypothesis on intimate interplay at the intestinal mucosa. Semin. Immunol. 19, 70-83

6. Muller, C. A., Autenrieth, I. B., and Peschel, A. (2005) Innate defenses of the intestinal epithelial barrier. Cell. Mol. Life Sci. 62 , $1297-1307$

7. Artis, D. (2008) Epithelial-cell recognition of commensal bacteria and maintenance of immune homeostasis in the gut. Nat. Rev. Immunol. 8, 411-420

8. Riccardi, C., Bruscoli, S., and Migliorati, G. (2002) Molecular mechanisms of immunomodulatory activity of glucocorticoids. Pharmacol. Res, 45, 361-368

9. Herold, M. J., McPherson, K G., and Reichardt, H. M. (2006) Glucocorticoids in T cell apoptosis and function. Cell. Mol. Life Sci. 63, 60-72

10. Schaaf, M. J., and Cidlowski, J. A. (2002) Molecular mechanisms of glucocorticoid action and resistance. J. Steroid Biochem. Mol. Biol. 83, 37-48

11. De Bosscher, K., Schmitz, M. L., Vanden Berghe, W., Plaisance, S., Fiers, W., and Haegeman, G. (1997) Glucocorticoid-mediated repression of nuclear factor-kappaB-dependent transcription involves direct interference with transactivation. Proc. Natl. Acad. Sci. U. S. A. 94, 13504-13509

12. Mukaida, N., Morita, M., Ishikawa, Y., Rice, N., Okamoto, S. Kasahara, T., and Matsushima, K. (1994) Novel mechanism of glucocorticoid-mediated gene repression. Nuclear factor-kappa $B$ is target for glucocorticoid-mediated interleukin 8 gene repression. J. Biol. Chem. 269, 13289-13295

13. Sapolsky, R. M. (2000) Stress hormones: good and bad. Neurobiol. Dis. 7, 540-542

14. Davies, E., and MacKenzie, S. M. (2003) Extra-adrenal production of corticosteroids. Clin. Exp. Pharmacol. Physiol. 30, 437-445

15. Cima, I., Corazza, N., Dick, B., Fuhrer, A., Herren, S., Jakob, S., Ayuni, E., Mueller, C, and Brunner, T. (2004) Intestinal epithelial cells synthesize glucocorticoids and regulate $T$ cell activation. J. Exp. Med. 200, 1635-1646

16. Mueller, M., Cima, I., Noti, M., Fuhrer, A., Jakob, S., Dubuquoy, L., Schoonjans, K., and Brunner, T. (2006) The nuclear receptor LRH-I critically regulates extra-adrenal glucocorticoid synthesis in the intestine. J. Exp. Med. 203, 2057-2062

17. Shinkai, Y., Rathbun, G., Lam, K. P., Oltz, E. M., Stewart, V. Mendelsohn, M., Charron, J., Datta, M., Young, F., Stall, A. M., and Alt, F. W. (1992) RAG-2-deficient mice lack mature lymphocytes owing to inability to initiate V(D) J rearrangement. Cell 68, 855-867

18. Coste, A., Dubuquoy, L., Barnouin, R., Annicotte, J. S., Magnier, B., Notti, M., Corazza, N., Antal, M. C., Metzger, D., Desreumaux, P., Brunner, T., Auwerx, J., and Schoonjans, K. (2007) LRH-1-mediated glucocorticoid synthesis in enterocytes protects against inflammatory bowel disease. Proc. Natl. Acad. Sci. U. S. A. 104, 13098-13103

19. Yamada, Y., Webber, E. M., Kirillowa, 1., Peschon, J. J., and Fausto, N. (1998) Analysis of liver regeneration in mice lacking type 1 or type 2 tumor necrosis factor receptor: requirement for type 1 but not type 2 receptor. Hepatology 28, 959-970 
20. Claassen, I., Van Rooijen, N., and Claassen, E. (1990) A new method for removal of mononuclear phagocytes from heterogeneous cell populations in vitro, using the liposome-mediated macrophage 'suicide' technique. J. Immunol. Methods 134, 153161

21. Van Rooijen, N. (1992) Liposome-mediated elimination of macrophages. Res. Immunol. 143, 215-219

22. Galanos, C., and Freudenberg, M. A. (1993) Mechanisms of endotoxin shock and endotoxin hypersensitivity. Immunobiology $187,346-356$

23. Wilson, N. S., Dixit, V., and Ashkenazi, A. (2009) Death receptor signal transducers: nodes of coordination in immune signaling networks. Nat. Immunol. 10, 348-355

24. Chen, G., and Goeddel, D. V. (2002) TNF-R1 signaling: a beautiful pathway. Science 296, 1634-1635

25. Xu, B., Yang, W. H., Gerin, I., Hu, C. D., Hammer, G. D., and Koenig, R. J. (2009) Dax-1 and steroid receptor RNA activator (SRA) function as transcriptional coactivators for steroidogenic factor 1 in steroidogenesis. Mol. Cell. Biol. 29, 1719-1734

26. Song, H. L., Lu, S., and Liu, P. (2005) Tumor necrosis factoralpha induces apoptosis of enterocytes in mice with fulminant hepatic failure. World J. Gastroenterol. 11, 3701-3709

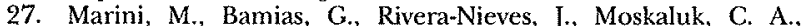
Hoang, S. B., Ross, W. G., Pizarro, T. T., and Cominelli, F. (2003) TNF-alpha neutralization ameliorates the severity of murine Crohn's-like ileitis by abrogation of intestinal epithelial cell apoptosis. Proc. Natl. Acad. Sci. U. S. A. 100, 8366-8371

28. Gitter, A. H., Bendfeldt, K., Schulzke, J. D., and Fromm, M. (2000) Leaks in the epithelial barrier caused by spontaneous and TNFalpha-induced single-cell apoptosis. FASEB J. 14, 1749-1753

29. Fries, W., Muja, C., Crisafulli, C., Costantino, G., Longo, G., Cuzzocrea, S., and Mazzon, E. (2008) Infliximab and etanercept are equally effective in reducing enterocyte APOPTOSIS in experimental colitis. Int. J. Med. Sci. 5, 169-180

30. Rutgeerts, P., Vermeire, S., and Van Assche, G. (2009) Biological therapies for inflammatory bowel diseases. Gastroenterology 136, $1182-1197$

31. Kassiotis, G., and Kollias, G. (2001) Uncoupling the proinflammatory from the immunosuppressive properties of tumor necrosis factor (TNF) at the $\mathrm{p} 55$ TNF receptor level: implications for pathogenesis and therapy of autoimmune demyelination. $J$. Exp. Med. 193, 427-434

32. Jacob, C. O., and McDevitt, H. O. (1988) Tumour necrosis factor-alpha in murine autoimmune 'lupus' nephritis. Nature $331,356-358$
33. Kontoyiannis, D., and Kollias, G. (2000) Accelerated autoimmunity and lupus nephritis in NZB mice with an engineered heterozygous deficiency in tumor necrosis factor. Eur. $/$. I mmu nol. 30, 2038-2047

34. Boivin, M. A., Ye, D., Kennedy, J. C., Al-Sadi, R., Shepela, C., and Ma, T.Y. (2007) Mechanism of glucocorticoid regulation of the intestinal tight junction barrier. Am. J. Physiol. Gastrointest. Liver Physiol. 292, G590-G598

35. Corazza, N., Eichenberger, S., Eugster, H. P., and Mueller, C. (1999) Nonlymphocyte-derived tumor necrosis factor is required for induction of colitis in recombination activating gene (RAG) 2(-/-) mice upon transfer of CD4(+) CD45RB(hi) T cells. J. Exp. Med. 190, 1479-1492

36. Matsuguchi, T., Musikacharoen, T., Ogawa, T., and Yoshikai, Y. (2000) Gene expressions of Toll-like receptor 2, but not Tolllike receptor 4 , is induced by LPS and inflammatory cytokines in mouse macrophages. J. Immunol. 165, 5767-5772

37. Abreu, M. T., Vora, P., Faure, E., Thomas, L. S., Arnold, E. T. and Arditi, M. (2001) Decreased expression of Toll-like receptor-4 and MD-2 correlates with intestinal epithelial cell protection against dysregulated proinflammatory gene expression in response to bacterial lipopolysaccharide. J. Immunol. 167, 1609 1616

38. Cario, E., Rosenberg, I. M., Brandwein, S. L., Beck, P. L., Reinecker, H. C., and Podolsky, D. K. (2000) Lipopolysaccharide activates distinct signaling pathways in intestinal epithelial cell lines expressing Toll-like receptors. J. Immunol. 164, 966972

39. Otte, J. M., Cario, E., and Podolsky, D. K. (2004) Mechanisms of cross hyporesponsiveness to Toll-like receptor bacterial ligands in intestinal epithelial cells. Gastroenterology 126, 1054-1070

40. Ortega-Cava, C. F., Ishihara, S., Rumi, M. A., Kawashima, K. Ishimura, N., Kazumori, H., Udagawa, J., Kadowaki, Y., and Kinoshita, Y. (2003) Strategic compartmentalization of Toll-like receptor 4 in the mouse gut. J. Immunol. 170, 3977-3985

41. Schenk, M., and Mueller, C. (2007) Adaptations of intestinal macrophages to an antigen-rich environment. Semin. Immunol. $19,84-93$

42. Bohan, A., Chen, W. S., Denson, L. A., Held, M. A., and Boyer, J. L. (2003) Tumor necrosis factor alpha-dependent up-regulation of Lrh-1 and Mrp3(Abcc3) reduces liver injury in obstructive cholestasis. J. Biol. Chem. 278, 36688-36698 